\title{
Using music as a signal for biofeedback
}

\author{
Ilias Bergstrom ${ }^{\mathrm{a}, \mathrm{b}, *}$, Sofia Seinfeld ${ }^{\mathrm{a}}$, Jorge Arroyo-Palacios ${ }^{\mathrm{b}}$, Mel Slater ${ }^{\mathrm{b}, \mathrm{c}}$, Maria V. Sanchez-Vives ${ }^{\mathrm{a}, \mathrm{c}}$ \\ a Institut de Investigacions Biomèdiques August Pi i Sunyer (IDIBAPS), Barcelona, Spain \\ b EVENT Lab, Facultat de Psicologia, Universitat de Barcelona, Barcelona, Spain \\ c Institució Catalana Recerca i Estudis Avançats (ICREA), Barcelona, Spain
}

\section{A R T I C L E I N F O}

\section{Article history:}

Received 12 July 2012

Received in revised form 10 April 2013

Accepted 16 April 2013

Available online $\mathrm{xxxx}$

\section{Keywords:}

Biofeedback

Music

Combined intervention

Arousal modulation

Sonification

\begin{abstract}
A B S T R A C T
Studies on the potential benefits of conveying biofeedback stimulus using a musical signal have appeared in recent years with the intent of harnessing the strong effects that music listening may have on subjects. While results are encouraging, the fundamental question has yet to be addressed, of how combined music and biofeedback compares to the already established use of either of these elements separately. This experiment, involving young adults $(\mathrm{N}=24)$, compared the effectiveness at modulating participants' states of physiological arousal of each of the following conditions: A) listening to pre-recorded music, B) sonification biofeedback of the heart rate, and $C$ ) an algorithmically modulated musical feedback signal conveying the subject's heart rate. Our hypothesis was that each of the conditions (A), (B) and (C) would differ from the other two in the extent to which it enables participants to increase and decrease their state of physiological arousal, with (C) being more effective than (B), and both more than (A). Several physiological measures and qualitative responses were recorded and analyzed. Results show that using musical biofeedback allowed participants to modulate their state of physiological arousal at least equally well as sonification biofeedback, and much better than just listening to music, as reflected in their heart rate measurements, controlling for respiration-rate. Our findings indicate that the known effects of music in modulating arousal can therefore be beneficially harnessed when designing a biofeedback protocol.
\end{abstract}

(c) 2013 Elsevier B.V. All rights reserved.

\section{Introduction}

Music's continuous, intensive, and multifaceted effect on listeners is extensively documented (Koelsch, 2010; Levitin and Tirovolas, 2009; Peretz, 2006). Numerous studies show several significant benefits of music used for arousal modulation towards therapeutic interventions, for a review see Nilsson (2009). Primarily it has been found to reduce the stress and anxiety experienced by patients in hospital care, through its facility to regulate mood and modulate arousal, and to serve as an effective analgesic, by distracting patients from the experience of pain.

Little research however has investigated the combined use of music with other technologically based interventions such as biofeedback. Our intention is to tackle this shortcoming, by investigating the benefits of using biofeedback and music in combination, towards modulating the level of arousal in healthy subjects. Our experiments were aimed at examining the possible effects arising from this combination, and to investigate the viability of music as a signal for biofeedback.

While research has contributed a significant body of knowledge on the effect of music compared to non-musical sound or silence, very little is known about how the effects of different types of music vary consistently across the human population, the universals of music. One clearly established universal effect is the direct correlation between the level

* Corresponding author at: IDIBAPS, Roselló 149-153, 08036 Barcelona, Spain. Tel.: + 34 $932275400 \times 4302$.

E-mail address: iliasbergstrom@ub.edu (I. Bergstrom). of arousal as represented through the tempo and volume of the music, and the level of arousal experienced by its listeners (Hunter and Schellenberg, 2010; Khalfa et al., 2002; Trappe, 2010; Zatorre, 2005). Besides the level of induced arousal, music universally also has the effect of entrainment on humans: the subconscious synchronization of various bio-rhythms to rhythms in the music. Besides entrainment on volitional movement (gait, tapping, head-nodding etc.) there are several reports on physiologically detectable effects on Autonomic Nervous System (ANS) responses (Clayton et al., 2005; Khalfa et al., 2008; Orini et al., 2010; Trappe, 2010). Direct entrainment effects have been observed on respiration rate (Haas et al., 1986), while Bernardi et al. (2009) demonstrated a clear synchronization between physiological measures (e.g., heart rate, respiration rate) and musical rhythm. These effects were observed across participants regardless of musical preference, with few differences in effect resulting from musical training. Further well established characteristics are that we are apt at perceiving fine variation and great detail in musical stimuli, and that music is particularly effective at drawing and maintaining our listening attention (Levitin, 2006). Using a range of physiological measurements, primarily cardiovascular and electrodermal, researchers have succeeded in differentiating between when subjects are listening to musical pieces of low and of high arousal (Hunter and Schellenberg, 2010).

The term music intervention refers to the use of music in a therapeutic context that is not supervised nor conducted by a trained music therapist; a much narrower context of application in comparison to music therapy, with demonstrated efficacy only in contexts where analgesic, anxiolytic 
and relaxing effects are desired (Nilsson, 2009). In the modern development of neurologic music therapy (NMT), knowledge about specific neurological mechanisms pertaining to music perception and production is harnessed, with application primarily in neurorehabilitation (De l' Etoile, 2010), for example in training gait control and speech production.

Commonly in biofeedback with sound, the feedback signal consists of a continuous tone of fixed timbre, whose frequency and/or amplitude are mapped to the underlying physiological measure, for example pitch increasing with chest expansion, or volume decreasing to reflect a decrease in tonic skin-conductance level. Single short tones commonly reflect events, for example heart-beats. Stereo panning or 3D audio may indicate the location of the conveyed parameter. For example, Chiari et al. (2005) use all three parameters for balance training: anteriorposterior acceleration modulates frequency, media-lateral acceleration modulates left-right balance, while both also control amplitude.

In established biofeedback practice, when physiological signals are conveyed sonically, as well as in auditory display, and more generally where information is conveyed through sound, the process involved is termed sonification: "the transformation of data relations into perceived relations in an acoustic signal for the purposes of facilitating communication or interpretation" (Kramer et al., 2010). In contrast, while music may also contain information as to the events or data that gave rise to it, music differs in intention: the emphasis is placed on the resulting sound itself, to be perceived and appraised for the listeners' aesthetic enjoyment (Hermann, 2008), while for sonification aesthetic enjoyment is a secondary concern to maximizing the ability of transparently reflecting data. Particularly relating to modern/atonal music, the distinction may not always be clear, since under composer Edgar Varèse's famous description of music as "organized sound" (Ross, 2007), sonified data also qualify as music. Under normal circumstances however the two are easily told apart.

The fact that both music interventions and biofeedback rely on analogous physiological processes, and that both interventions are used to treat similar conditions, leads to asking what benefits may be drawn from their combined use. The fact that musical stimuli have such universally powerful effects on listeners may be very positive in biofeedback if these effects are accounted for, but also very detrimental if not. Indeed it has been stated that much research is needed to examine how the combined use of biofeedback and music may be effectively accomplished, as little systematic work has been carried on this to date (Ellis and Thayer, 2010).

Few experimental studies report explicit use of a musical feedback signal. For alpha activity neurofeedback training, van Boxtel et al. (2012) used participant-selected music, relying on music's ability to attract and maintain attention, and to entertain participants. In the context of biofeedback studies, Liu (2010) details his successful application of a HR biofeedback based in-flight music recommendation system, for regulating the arousal of long-haul flight passengers. Cui et al. (2010) present a multimodal biofeedback system for regulating the respiration during computer tomography, for eliminating artifacts in the captured image caused by irregular breathing. Chen et al. (2009) described two musical and visual biofeedback protocols for training stroke patients in reaching and grasping an object, both using the same predefined music across participants. Yokoyama et al. (2002) presented a biofeedback system where music was controlled using the heart rate (HR) of a subject, and evaluated the system's usefulness towards relaxing the subject during office work.

We have not identified any studies preceding ours that directly compare their music biofeedback protocols to biofeedback protocols that do not use music as the feedback signal, or to the effects of music alone. It thus remains an empirical question as to whether listening to music in the context of a biofeedback paradigm elicits more effective (stronger) changes than either technique alone. Hence, this research directly addresses this issue, through an experiment comparing the effectiveness at modulating the participants' state of physiological arousal following each of three conditions. Besides answering the above hypothesis, we also set out to learn and report what design considerations are important in devising a musical biofeedback protocol.

In the main experiment reported, we observed the participants' responses to such a combined stimulus, by measuring physiological responses of participants instructed to arouse and relax as much as they could under three different conditions: A) while either listening to arousing or relaxing music, B) a sonification of their heart rate using a sine-wave varying in pitch, or C) the previously used arousing and relaxing music, the tempo and volume of which was controlled by the subject's heart rate (which we've christened combined biofeedback). Higher HR increased musical tempo (with pitch remaining stable) and amplitude, while lower HR resulted in slower tempo and lower amplitude. Our hypothesis was that each of the conditions (A), (B) and (C) in order, would be increasingly better than the previous two in the magnitude to which it enabled participants to increase and decrease their state of physiological arousal: $(\mathrm{C})>(\mathrm{B})>($ A). Besides quantitative physiological measurements, we also gathered qualitative responses using questionnaires, on the experience, musical ability, and on participant's body perception awareness.

\section{Methods}

\subsection{Participants}

Participants (18 females, 6 males, $\mathrm{M}_{\mathrm{age}}=28.2$ years, $\mathrm{SD}=6.3$, age range: 19-44 years) were recruited over email or from around University of Barcelona's Psychology campus. Participants were compensated with $6 €$ for their participation. Focusing on the musical experience of the sample, sixteen of the participants had attended music lessons, three had formal music studies, while only three identified themselves as being amateur musicians or better. Twenty participants reported that they listen to music daily, with the reported motivation for listening to music having a median value of 4.0 , IQR (interquartile range) $=0$ on a $1-5$ Likert type scale ( 1 being graded as not motivated at all, 3 neither a little nor a lot, and 5 extremely motivated). Chills from listening to music were reportedly experienced by twenty one participants, and while twenty three reported that music alters their emotional state, all reported that their emotional state varies depending on the musical genre listened to. For safety reasons, pregnant women, people with epilepsy or with cardiovascular problems were excluded, and we also excluded people that reported severe uncorrected auditory and visual problems. Upon arrival at the laboratory all participants were asked to read and sign a consent form. The study was carried out in accordance with the regulations of the Comisión de Bioética de la Universitat de Barcelona, and was therefore performed in accordance with the ethical standards laid down in the 1964 Declaration of Helsinki.

\subsection{Materials}

\subsubsection{Musical biofeedback system overview}

For our protocol we emphasized controlling the known universal effects of music to modulate level of arousal, and to draw attention without distracting from the biofeedback training. Optimal enjoyment, albeit considered important, was given less priority. We decided to convey the physiological state of participants by controlling musical tempo and volume, since past studies have shown that these parameters are capable of modulating changes in arousal (Khalfa et al., 2002; Zatorre, 2005; Hunter and Schellenberg, 2010; Trappe, 2010).

To determine the design of the experiment and of the biofeedback system and protocol to be used, we carried out two pilot experiments. This experience helped us pick whether heart rate (HR) or Galvanic Skin Response (GSR) was the most suitable physiological measurement to use as a feedback signal. We observed HR to vary as desired, mostly contained within $\mathrm{a} \pm 20 \mathrm{BPM}$ range, while for GSR, participants showed comparatively few changes and low variability. This might be due to 
individual response stereotypy and some participants having more electrodermal lability or stability (Dawson et al., 2000). In addition, HR responses reflect changes in sympathetic and parasympathetic activity, while electrodermal responses are only modulated by the first system. For the purpose of this experiment, we thus considered HR to be a more appropriate feedback signal. Finally, during pilots we found that the largest differences between conditions appeared over a three-minute window, and thus decided to use this duration for tasks in the final experiment.

Musical biofeedback presents new challenges when compared to visual or sonification biofeedback. Importantly, the ranges of tempo and volume change that can meaningfully be manipulated are limited, by human perception, as well as by what is musically meaningful. While most healthy people can visually perceive a 1-2 pixel movement of a graphical element on a computer display, few would be able to perceive a tempo change of less than 2-3 BPM, or an analogously small amplitude change (Thomas, 2007). While tempo changes are common in music, the extent of change within a single composition is much smaller than the full range of possible musical tempi. We are not aware of any absolute guidelines governing tempo change extent, and surely this extent varies greatly depending on musical genre. Still, care has to be taken to define these ranges for the specific music used, to avoid a decidedly unpleasant signal. The implication of the above for biofeedback protocol design, is that it is required to precisely pre-determine the ranges used in the mapping: the range of values to be interpolated from in the physiological measurements, and the ranges of musical tempo and volume these are interpolated to, so as to maximize the effective resolution of the biofeedback system.

For the pieces used in this experiment, following our pilot experiments we subjectively determined the tempo range to be within $\pm 20 \mathrm{BPM}$, while setting the minimum and maximum amplitude levels to be well within the ranges of what young healthy participants can perceive. The HR measure was determined by a moving window over the last four beat intervals, since instantaneous rate feedback was found to be too abrupt, giving rise to un-musical changes in the feedback signal, while a window of four made for a good compromise between fast feedback, and maintaining a musical feedback signal. We used linear interpolation to map values between the ranges of HR and tempo.

For the reproduction of real-time manipulated music and sound, we implemented a custom plug-in specifically for this experiment in the Max/MSP (Max/MSP, 2012) programming environment, executed using the Ableton Live 8 (Ableton, 2012) music sequencing application as a host. For the real-time tempo time-stretching of the music, the built-in capabilities of Live 8 were harnessed through their real-time automation, controlled by our custom plug-in. For time-stretching, Live 8 uses the élastique Pro V2.0 algorithm, which Ableton has licensed from zplane (zplane, 2011). It allows the time-stretching of recorded music by $\pm 20 \%$, without producing audible artifacts. Our custom plugin received the physiological measurement data from the electrophysiological monitoring devices detailed in Section 2.2.3, over a UDP network connection, with all software being executed on the same Windows 7 PC laptop. Music was played through a pair of Beyerdynamic DT-770M headphones, driven by an external Roland UA-25EX audio interface.

\subsubsection{Music for biofeedback}

For iteratively generating new musical pieces to use in our feedback protocols, a process informed by our advancing knowledge and experience on what compositional characteristics were required, we took advantage of the music composition flexibility facilitated by the Melomics project's algorithmic composition software (Diaz-Jerez, 2011; Melomics, 2012), using which we could instantaneously access custom musical pieces, created to our specification.

We decided to use the same compositions across participants. The two musical pieces used for the experiment were both composed within the Melomics software, with parameters specified to satisfy detailed composition criteria for the intended purpose, resulting from an exhaustive review of the literature on music perception (e.g. Ball, 2010; Sloboda, 2005), and through pilot experiments.

The musical pieces used are both available online for readers to listen to, in the paper's accompanying material on the publisher's website. They were carefully composed to avoid giving rise to undesirable feelings and associations in participants, by using a neutral, classical orchestration, and not including lyrical content. For the same reason, we avoided obvious similarities to popular existing compositions that could give rise to strong associations. Both pieces were composed to cover the frequency spectrum, and not be perceived as primarily high, or primarily low in their spectral composition and width. For the relaxing piece, we opted to include clear rhythmical events, towards facilitating rhythm entrainment, while still ensuring that these do not counteract the relaxing character of the piece. The composition was slow-changing, to avoid arousal responses from unexpected events (Salimpoor et al., 2011), albeit not being entirely static, so as to maintain the participant's interest. The tempo was made to be perceived as being 60 BPM, a tempo effective for relaxation music interventions (Nilsson, 2008). For the activating music, the composition was distinctly rhythmical, providing a clear beat to entrain to. We maintained a high density of compositional changes where these made musical sense, to elicit consistently sustained arousal responses. Instrumentation was dense and varied. The tempo was made to be perceived as being 140 BPM. Finally, most instruments were densely active, to maximize the arousing effect achievable with the music, and avoid ambiguity in the perception of the music's tempo.

\subsubsection{Physiological monitoring devices}

The physiological data was captured using the g.MOBIlab + device (g.tec, Guger Technologies OEG, Graz, Austria). Bipolar ECG was measured by placing three electrodes on the left and right collarbones and the lowest left rib of each participant. Furthermore, a piezo-crystal respiration effort sensor from SleepSense (SleepSense, 2012) was placed on the upper part of the chest to record respiratory measures. The g.MOBIlab + was integrated into a real-time system using Simulink (Simulink, 2012) to process and store physiological data at a sample rate of $256 \mathrm{~Hz}$. The Simulink model processes the raw signals from the sensors and calculates in real-time measures such as HR and respiration rate. We used the g.MOBIlab + for applying a $50 \mathrm{~Hz}$ notch filter to each of the analog channels of the device for suppressing line noise interference. The g.MOBIlab + sends data to the PC with the Simulink model via Bluetooth, while the Simulink model communicates with our biofeedback software (detailed in Section 2.2.1) via UDP.

\subsection{Measurements}

\subsubsection{Heart rate}

The main measurement of this experiment was heart rate (HR) measured in beats per minute (BPM), given as it was the physiological measure used to control the feedback signal. The electrocardiogram data was post-processed off-line and manually corrected to ensure the elimination of false-positive and false-negative heart beats prior to analysis, using the g.BSanalyze biosignal analysis package with the ECG toolbox extension (g.tec - Guger Technologies OEG, Graz, Austria). The process entailed the automatic detection of QRS (ventricular contraction) complexes in the ECG time series based on a modified Pan-Tompkins algorithm (Pan and Tompkins, 1985). Subsequently, a visual inspection of the QSR detected was carried out to correct missing or wrongly assigned points. Following this method we determined the distance in time from one heart contraction to the next (RR interval).

\subsubsection{Respiration rate}

The respiration rate measures the inhalation and exhalation phases of the participant. We decided to include this measurement since 
respiration is under participants' volitional control, and may have been used as a direct strategy to influence HR. It is measured in units of respirations per minute throughout this manuscript. The respiration rate of participants was also post-processed off-line, with individual respiration cycles being automatically detected, and subsequently also manually corrected to eliminate false-positive and false-negative cycles, using the g.BSanalyze package.

\subsubsection{Awareness sub-scale of Body Perception Questionnaire (BPQ)}

We were unable to find reports on the development and validation of the entirety of the Body Perception Questionnaire (Porges, 1993). The complete version of the questionnaire includes five subscales related to stress and perception of bodily responses. The awareness subscale of the BPQ however has in past studies been demonstrated to be a useful measure of self-rated bodily awareness (Critchley et al., 2004; Mehling et al., 2009), and was here used to assess possible differences in interoceptive awareness between participants.

\subsubsection{Questionnaires created specifically for this experiment}

Towards gathering demographic information, data on participants' musical experience and preferences, and subjective feedback on the experiment experience, we had to create suitable questionnaires ourselves, since no appropriate pre-existing questionnaires could be located that would suit our needs. The questionnaires we created are available to read in Appendix B of this article.

\subsection{Procedure}

\subsubsection{Experiment design}

The effectiveness for modulating the participant's state of physiological arousal of each of the following three conditions was compared: A) listening to pre-recorded music, B) sonification biofeedback, and C) combined biofeedback, where the feedback signal was algorithmically modulated music. Each such condition consisted of a baseline measure of 3 min followed by two tasks: 1) 3 min for achieving the maximum level of the participant's arousal, and 2) 3 min for achieving the minimum level of the participant's arousal.

Our hypothesis was that each of the conditions (A), (B) and (C) would differ from the other two in the extent to which they would enable participants to increase and decrease their state of physiological arousal, with (C) being more effective than (B), and both more than (A). The following are detailed descriptions of each condition in turn.

A) First, the initial baseline measurement was carried out. In random order, participants then listened to either a piece of music composed to be as arousing as possible or as relaxing as possible. For each, they were instructed to augment their state of physiological activation, to either as aroused or as relaxed as possible, while paying attention to the arousing or relaxing music played. This is what we for the purpose of this article refer to as (A), the music condition.

B) Again, first the initial baseline measurement was carried out. Participants then listened to a sine-tone sound of which the pitch was in real-time altered to reflect the participant's HR. In random order, they were instructed to either increase the tone's frequency as much as they could by augmenting their level of physiological arousal, or decrease the frequency as much as they can through relaxing. This is what we for the purpose of this article refer to as (B), the biofeedback condition.

C) Again, first the initial baseline measurement was carried out. Subsequently, in random order, participants listened to either arousing or relaxing music. They were instructed that the volume and tempo of the music would reflect their state of physiological arousal, and that they should either make the music as fast and loud as they can, or as slow and soft, depending on the condition. The physiological signal used to modulate the tempo and volume of the musical pieces was also in this case HR. This is what we for the purpose of this article refer to as (C), the combined biofeedback condition.

Each participant completed 6 trials in counterbalanced order across the two factors of condition and task. Before the two condition tasks, the baseline measurement always took place, while tasks 1 \& 2 were randomized in order between participants. The random assignment of participants to groups G1-G12 (see Table C.1 in Appendix C) was carried out using the "Research Randomizer" web tool (Urbaniak and Plous, 2011), ensuring that each order of conditions was executed the same number of times as the other orders.

In both the music and the combined biofeedback conditions, the arousing and relaxing musical compositions used were the same. In both the biofeedback and the combined biofeedback condition, the physiological signal used to control the feedback was that of HR, but in neither condition were the participants explicitly told of this, instead they were only told that the feedback stimulus reflects their overall physiological arousal.

\subsubsection{Experimental procedure}

In all conditions participants were seated in a quiet, temperatureregulated room, in a comfortable chair, wearing headphones during all tasks except for the baseline ones. Participants were given a document to read with a full description of the experiment, and were allowed to ask clarifying questions, prior to being given a consent form, which they only signed if they agreed to take part in the experiment.

In the baseline measurements, participants were seated in silence, and were instructed to remain still, with their eyes open, not paying particular attention to any events in their surroundings, or any thought entering their mind. At the beginning of each condition, participants were verbally instructed of what the condition would entail, while at the beginning of each task, participants were signaled with a written sign instructing them on what to do in the immediately following task. When each condition was over, participants were made aware of the fact through the sound stimulus immediately going quiet. Participants were instructed to try and remain still during all the above conditions, particularly avoiding hand, finger and leg movements. When instructed to alter their state of physiological arousal, no specific instructions or training were given as to how this may be achieved, nor were any instructions given for participants to not use any training they may have towards achieving such a goal. During the first baseline measurement, the mean HR was observed, and used as input for the real-time linear interpolation algorithm of the music: +/-20 BPM in participant HR was mapped to a $+/-20 \%$ change in the music's tempo, relative to the base tempo for which each piece was composed.

After each 9 minute sequence of three 3-minute tasks, participants were able to rest before the start of the next sequence. The total duration of the experiment conditions was thus 27 min per participant, with an equal amount of time necessary for setting up the equipment, instructing the participant, and administering the questionnaires, leading to an approximate total engagement of $1 \mathrm{~h}$ for each participant. Before the experimental conditions we administered demographic questionnaires, while after the experience we administered the subjective experience and body perception awareness questionnaire, detailed in Sections 2.3.3 and 2.3.4.

The musical pieces used are both available online for readers to listen to, in the paper's accompanying material on the publisher's website (Sound examples $1 \& 2$ ). These files also serve as examples of conditions A-1 \& A-2. Sound examples 3 \& 4 are simulations of the biofeedback conditions B-1 \& B-2, while Sound examples 5 \& 6 are simulations of the combined music biofeedback conditions C-1 \& C-2. Note that in all cases the sound is not from an actual experiment participant, but a simulation of what that might have sounded like. 
Table 1

Participants' ratings of each experiment condition's effectiveness on a Likert scale of 1-5.

\begin{tabular}{ll}
\hline Condition & Median \\
\hline Music up & 3.0 (IQR 0.5) \\
Music down & 3.0 (IQR 1.0) \\
Biofeedback up & 4.0 (IQR 1.0) \\
Biofeedback down & 4.0 (IQR 2.0) \\
Combined up & 3.5 (IQR 1.0) \\
Combined down & 4.0 (IQR 1.25) \\
\hline
\end{tabular}

\subsection{Statistical treatment of the data}

For questionnaire data we used non-parametric tests since the scores are ordinal. For all physiological measures we used standard ANOVA including a test to check that the residual errors of the fit satisfied normality using the Shapiro-Wilk test. In one case outliers were removed to satisfy normality, and in another case a simple transformation to a log scale sufficed. Finally, we carried out a path analysis to further understand the dependencies among the variables included in the study. The Stata 12 program was used for all analysis.

\section{Results}

Participants' median subjective rating of each musical composition's effectiveness for its intended purpose on a 1-5 Likert scale, where 0 meant not effective at all, and 5 meant extremely effective, was 3 IQR 2.0 for the arousing music, and 4 IQR 0.25 for the relaxing music. No significant differences were found following a sign test, between the reported subjective perceived control over the sonification (median 3 IQR 2.0) and music biofeedback (median 2 IQR 1.25) signals, $P=0.2$. Table 1 details participants' subjective ratings on the perceived effectiveness of each experiment condition at modulating their level of arousal. No significant differences were found between these. No significant differences between groups were found when analyzing demographic, body perception awareness, or any of the other elicited questionnaire responses (Appendix B.2).

For heart rate measures (see Fig. 1) we refer to HRB as the heart rate in the baseline condition and HR as the heart rate in the experimental conditions (Task: Up or Down).

There is no difference between the mean heart rates across the 6 cells of the factorial design. The min and max mean HRB are $70.1 \pm 8.1 \mathrm{BPM}$ and $71.3 \pm 8.0 \mathrm{BPM}$ (see bracketed figures in Table 2).

It is clear from Table 2 that there is no effect of condition on HR.
Two-way ANOVA was carried out using HRB as a covariate (to remove the influence of differences between individuals). This resulted in significance only for Task $(\mathrm{F}(1,137)=24.7, \mathrm{P}<0.00005$, partial $\left.\eta^{2}=0.15\right)$, showing that HR was greater for 'Task Up' than for 'Task Down'. Nothing else was significant including the interaction term. However, analysis of the residual errors of the fit showed that these were not normally distributed (Shapiro-Wilk test $\mathrm{P}<0.00005$ ). Inspection of the residual error plot revealed 4 outliers. When these were removed the residual errors satisfy normality ( $S W$ test $P=0.18$ ), but the results do not change - only task is significant $(F(1,133)=$ 26.9, $\mathrm{P}<0.00005$, partial $\eta^{2}=0.17$ ).

Apparently then the instruction to change heart rate was effective independently of the method used to achieve this.

We next considered respiration rate (Resp) and respiration rate in the baseline condition (RespB), see Fig. 2 .

Table 3 shows again that task had an influence on respiration rate, and possibly also condition had an influence.

ANOVA of Resp with RespB as a covariate also results in nonnormally distributed errors but here transforming both to a log scale removes this problem, hence we use LResp $=\log (\operatorname{Resp})$ and LRespB $=\log (\operatorname{RespB})$. Here we find again task to be significant $\left(F(1,137)=93.8, P<0.00005\right.$, partial $\left.\eta^{2}=0.41\right)$, the main effect of condition is not significant $(P=0.78)$ but the interaction term is significant $\left(F(2,137)=3.46, P=0.03\right.$, partial $\eta^{2}=0.05$ ) (ShapiroWilk, $\mathrm{P}=0.45)$.

Fig. 3 shows the predicted means under the combinations of condition and task. As would be anticipated from the above significant interaction term, the slopes are different and in line with what we would expect. Music biofeedback shows the greatest change and music alone the least. However, it can also be seen from the confidence intervals that there is not a significant separation between the means, as is also reflected by the non-significant main effect of condition, though the trend suggests that respiration rate was important in these results.

There are two reasons for considering respiration rate. First it is known that respiration rate modulates heart rate (Holmes et al., 1980; Song and Lehrer, 2003), and this is also illustrated here in Fig. 4 (the same relation holds between HRB and RespB). Additionally participants may have deliberately tried to control their heart rate through a respiration strategy - breathing at greater frequency to increase HR and at lesser frequency to lower it.

Hence in attempting to understand the impact of the various conditions and tasks on HR we had to control for respiration rate. This is possible using path analysis (Kaplan, 2008). The path method supports analysis of a set of simultaneous equations over the

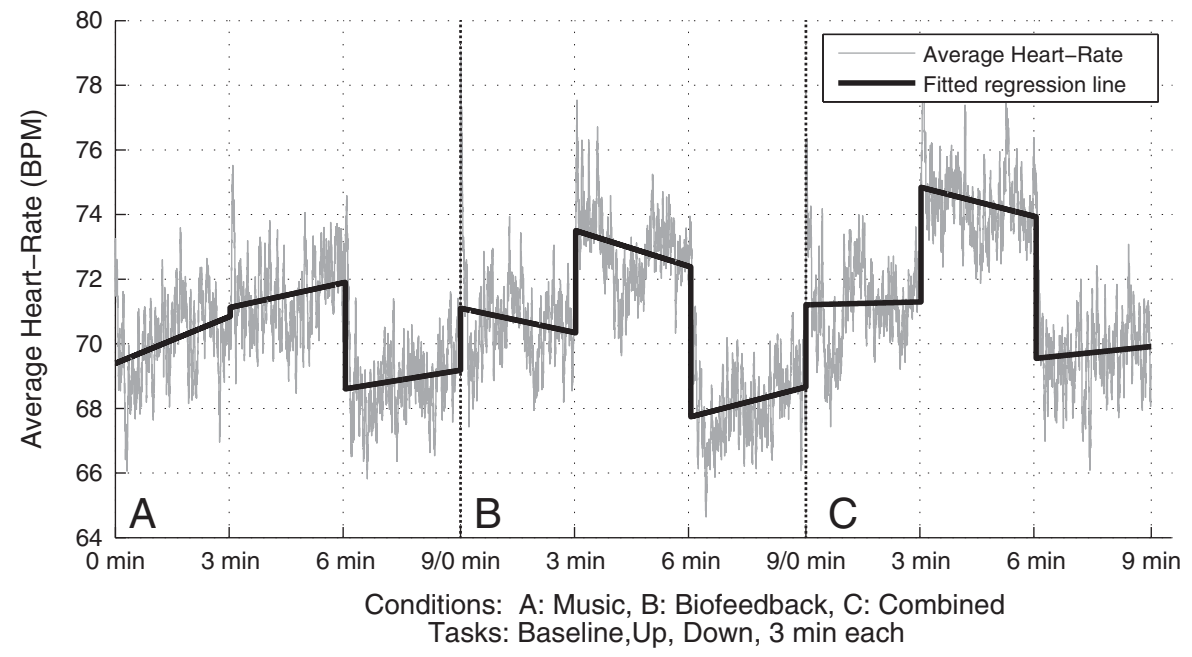

Fig. 1. Averaged heart rate for condition A, B \& C, each graph containing baseline, arousal and relaxation tasks for its respective condition, across all participants. 
Table 2

Mean \pm SD of heart rate beats per minute in the experimental conditions. $n=24$ per cell (mean HR baseline shown in brackets).

\begin{tabular}{lll}
\hline Condition & Task Up & Task Down \\
\hline Music & $71.5 \pm 6.4$ & $68.9 \pm 8.2$ \\
& $(70.1)$ & $(70.1)$ \\
Biofeedback & $73.0 \pm 9.2$ & $68.2 \pm 8.7$ \\
Music biofeedback & $(70.7)$ & $(70.7)$ \\
& $74.4 \pm 9.0$ & $69.7 \pm 8.2$ \\
& $(71.3)$ & $(71.3)$ \\
\hline
\end{tabular}

variables, rather than treating each equation separately. Path analysis was carried out using the Structural Equation Modeling software of Stata 12. Estimates and significance levels were computed using the asymptotic distribution free option, which does not rely on underlying multivariate normal distributional assumptions.

The path model (Fig. 5) is based on simple assumptions - that Task (Up or Down) may affect both respiration and heart rate. Moreover, when considering HR, the effect of HRB, Resp and RespB should be taken into account, and when considering Resp, RespB should be taken into account. The path analysis is run over the three conditions.

The results are shown in detail in Appendix A, Table A.1 (variances are not shown). The interpretation of this table is quite straightforward, and the goodness of fit test (Chi-squared) suggests a good overall fit. The most important variable is HR. Let's consider HR with condition $=2$ (biofeedback). The fitted equation is:

$\mathrm{HR}=-0.9-2.2 * \mathrm{LResp}+0.9 * \mathrm{HRB}-5.7_{\text {(if Task }=\text { Down })}+7.9 * \mathrm{LRespB}$

The standard errors, $\mathrm{z}$ values for significance and the corresponding $P$ values are shown.

The most important coefficients are those for task in the equation for HR. Here the results show that for the Task Down, this results in a decrease in fitted HR. However, conditions 2 and 3 (both biofeedback conditions) are highly significantly different from 0 whereas condition (music only) is not. Recall that this is also controlling for the effect of respiration.

These results suggest that there was a differential effect of condition on both respiration and more importantly heart rate after controlling for respiration. The biofeedback conditions had a greater effect than simply playing music. However, the results cannot distinguish between the effects of biofeedback using a tone and biofeedback using the music.
Table 3

Mean \pm SD of respiration rate (respirations per minute) in the experimental conditions. $\mathrm{n}=24$ per cell (mean respiration rate baseline shown in brackets).

\begin{tabular}{lll}
\hline Condition & Task Up & Task Down \\
\hline Music & $24.9 \pm 12.4$ & $18.4 \pm 10.7$ \\
Biofeedback & $(19.4)$ & $(19.4)$ \\
Music biofeedback & $25.5 \pm 12.1$ & $17.2 \pm 11.3$ \\
& $(18.5)$ & $(18.5)$ \\
& $28.4 \pm 11.9$ & $16.6 \pm 12.1$ \\
\hline
\end{tabular}

\section{Discussion}

Our results demonstrate that employing a combined music and $\mathrm{HR}$ biofeedback protocol to modulate arousal (condition $\mathrm{C}$ ) is more effective than instructing participants to themselves modulate arousal while listening to arousing and relaxing music respectively (condition A), and comparable to using a pure sonification biofeedback protocol (condition B). We observed that respiration rate (Fig. 2, Table 3 ) has probably been employed by participants as a means of adjusting their level of arousal, increasing the breath rate to arouse and decreasing the breath rate to relax. Note that this was without us having given any instruction to the participants of what they should do to achieve these states, either by modulating their respiration rate or otherwise. Still, our path analysis shows that when controlling for respiration and its modulatory effects, there is a remaining and significant influence of task on heart rate.

From our experience with this study, we have realized the importance of choosing whether to use participant-selected music, or to use the same compositions across participants, selected by the experimenters, when designing a protocol for music biofeedback. A middleground may sometimes also be suitable, where participants select from a database, or experimenters pick from a selection provided by the participant. We have seen all three approaches in our review of previous studies (see below). Choosing an approach is necessarily an informed compromise, dependent on the effects of music that are desirable in the particular protocol: is it the universals of arousal modulation and entrainment, is it the attention-drawing effect of music, is it mood regulation, or is it to entertain the participant, thus maintaining active engagement in the protocol? In protocols relying on participantselected music it is harder to closely control the effects of music's universals, since the composition varies greatly between participants. In the worst case the risk is that a participant-selected song is wholly unsuitable for the protocol intended, as it may for example arouse the

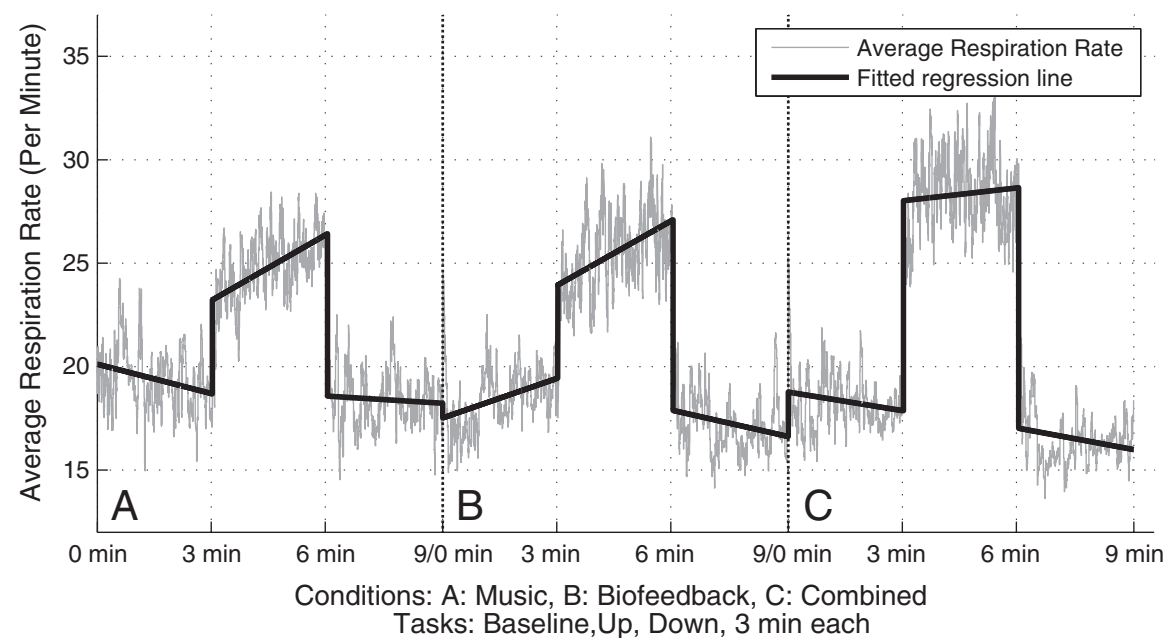

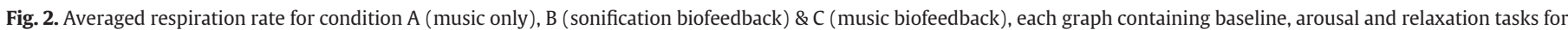
its respective condition, across all participants. 


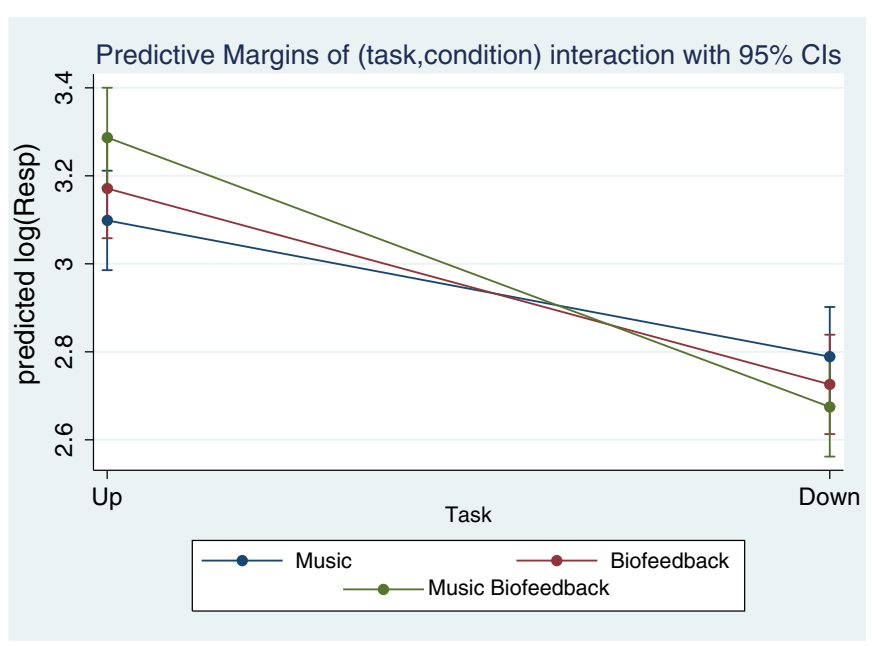

Fig. 3. Margin plot for Log Respiration Rate with 95\% (non-simultaneous) confidence intervals.

participant when the intention is relaxation, or be too distracting from the protocol at hand. Protocols that use the same compositions across all participants, can better control the known universal effects of music, and ensure that all participants have as similar as possible an experience. On the other hand the composition needs to be carefully selected so as to be pleasant listening for the majority of participants, and intense pleasure experiences such as chills down the spine (Levitin and Tirovolas, 2009) are unlikely to occur. Any music selection procedure thus necessarily requires consideration before deciding on the approach that best suits the protocol at hand.

Towards the iterative definition of the musical compositions to be used, a novel approach in the context of musical biofeedback is to employ algorithmic composition, as demonstrated by our use of the Melomics software. Having the ability to instantaneously access custom musical pieces that match our detailed criteria made the music selection process much easier than resorting to the alternatives, of searching for matching existing compositions every time we revised our requirements, or hiring a composer to iteratively compose new music.

An important caveat derived from the research experience stemming from this study, is that the potential benefits of devising a combined music biofeedback protocol are not guaranteed, as the successful outcome of its application depends on careful balancing of several design choices in the protocol. We have furthermore not been able to point

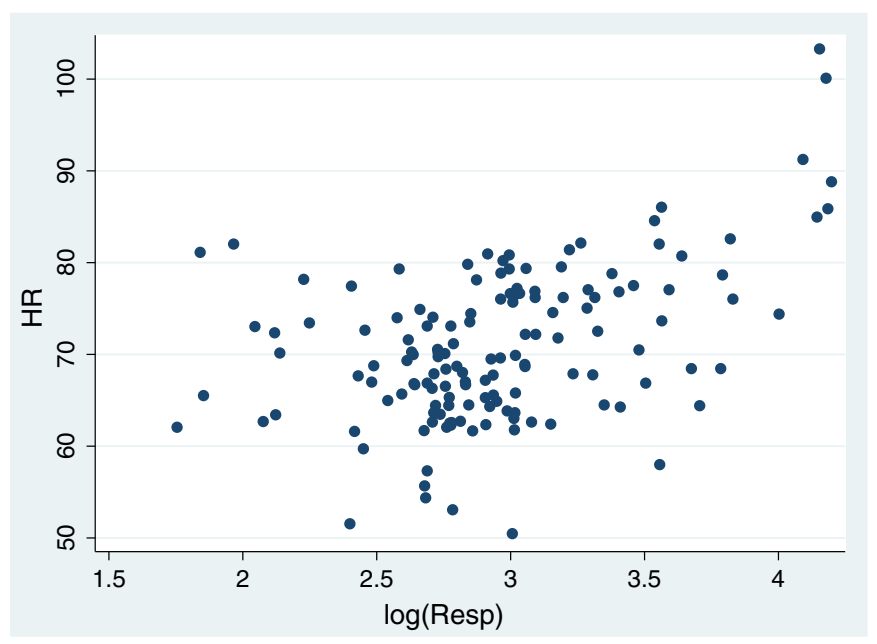

Fig. 4. Scatter diagram of heart rate (HR) on Log Respiration Rate $\log ($ Resp) all conditions and tasks included. ( $\mathrm{R} 2=0.22, \mathrm{P}<0.0005$ ). to differences in effect between the sonification biofeedback (B) and combined music biofeedback (C) conditions. We hypothesize this is likely a side effect of our chosen experiment protocol, but we cannot rule out that it could also be because there is no benefit in choosing music biofeedback over a sonification biofeedback protocol. The task length of only 3 min might be part of the reason for why we saw no differences, and a study using tasks lasting 10-15 min or longer, could have potentially had a different outcome. It is crucial to point out how biofeedback protocols of longer duration are naturally made far more feasible with music biofeedback, since music listening is a task that people naturally devote long periods of time to, as opposed to the potentially much less fulfilling task of listening to a sonification or observing a graph.

The lack of previous research on comparing a combined musical biofeedback protocol to each of its constituting elements was recently pointed out by Ellis and Thayer (2010). Results such as those derived from our study, can by extension also lend stronger support to studies that employ combined music biofeedback protocols, both past (Chen et al., 2009; Cui et al., 2010; Liu, 2010; Yokoyama et al., 2002) as well as future studies. It was previously known that the feedback stimulus used plays a very significant role in determining the outcome of the feedback protocol. In the context of sonification neurofeedback, Tyson (1982) showed that as simple a variation as using saw-tooth or sine-wave tones as a feedback signal can result in dramatically varied outcomes. Tyson's result shows that in a biofeedback protocol subjects not only perceive a signal that represents different physiological parameters but also interpret the feedback stimulus in a cognitive and affective way, as is also supported by Schater and Singer's (1962) two-factor theory of emotion. Olson (2003) stresses that an important feature of a biofeedback technique is that the signal should have reinforcing properties, for helping participants in learning how to gain control over their physiological states. Building on this knowledge it is straightforward to imagine the many ways in which musical characteristics may also negatively influence the outcome of the biofeedback training, for example if music listening induces arousal when the intention is relaxation, or if music gives rise to associations which distract from the training task at hand.

We have in previous studies observed that in some cases the feedback protocol used does not in fact constitute a continuously optimal musical experience, withholding aspects of the musical stimulus till the end, to be provided as rewards upon achieving the goals of the protocol. Furthermore we have observed that while reviewed protocols often make ingenious use of the musical stimulus, the design considerations and decisions behind their implementation are not always explicitly detailed, or discussed in the light of possible alternatives. We have for the present study made sure to directly show how we reached these decisions in comparison to possible alternative options, so as to inform future researchers when they are faced with similar biofeedback protocol design decisions.

van Boxtel et al. (2012) used participant-selected music, relying on music's ability to attract and maintain attention, and to entertain participants. The controlled variable was the cut-off frequency of a high-pass filter: a higher cut-off removed more of the musical signal. We believe the design's drawback is that as the cut-off point increases, so does the signal's unpleasantness: musical content is increasingly removed from a composition familiar to listeners, as if simulating a low quality music reproduction system incapable of reproducing frequencies that the listener expects to hear. Employing such a protocol with user-selected music risks resulting in the music functioning as reward, the signal during the protocol serving more as anticipation. Commendably, van Boxtel et al. used risky hypothesis testing, their control consisting not only of placebo music without feedback, but also the feedback protocol using random beta spectrum brainwaves instead of alpha. While they confirmed that alpha feedback training more effectively relaxes participants than random beta training, the benefit of using musical feedback remained unexamined. Furthermore, we argue that a protocol that ensures the musical stimulus is 


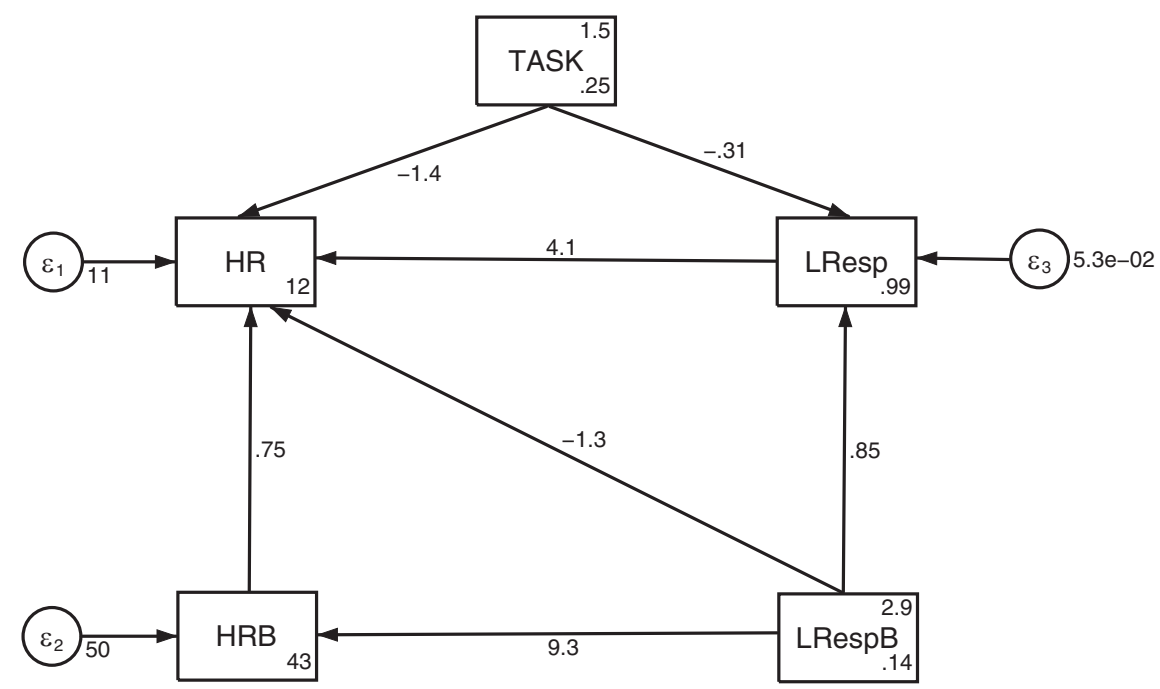

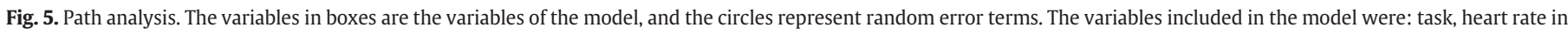

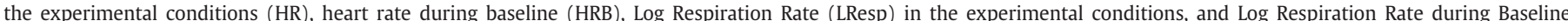

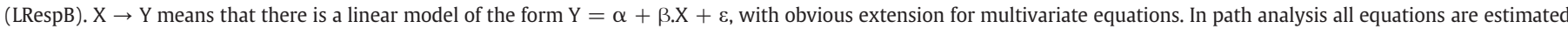

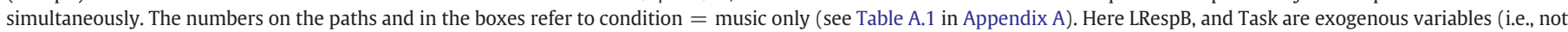
determined within the model). Task is coded as UP $=1$ and Down $=2$. The values on the paths from the circles are variance estimates.

continuously as pleasant as can be, may have shown benefits over the feedback signal van Boxtel et al. employed.

Switching the focus to biofeedback studies, Liu (2010) details his successful application of a HR biofeedback based in-flight music recommendation system, for regulating the arousal of long-haul flight passengers. Their protocol is a good example of allowing participants to select their own set of music, from which a song with the desired characteristics is automatically picked. Their protocol is for use over several hours, removing the need for real-time algorithmic manipulation of the music, instead adapting the feedback through varying song selection. Cui et al. (2010) present a multimodal biofeedback system for regulating the respiration during computer tomography, for eliminating artifacts in the captured image caused by irregular breathing. The music was specifically created for the experiment, and stable across participants. Their feedback protocol exemplifies effective harnessing of known characteristics of human musical perception: music's entraining ability, and musical perception's fine temporal acuity. It involved two musical signals, one at constant tempo reflecting a target breathing rate, and another whose tempo varied to reflect actual breathing rate. Participants then learned to stabilize their breathing rate by maintaining the two musical phrases in synch. Chen et al. (2009) described two musical and visual biofeedback protocols for training stroke patients in reaching and grasping an object, both using the same predefined music across participants. Analogously to van Boxtel et al. (2012), the feedback did not continuously manipulate musical parameters towards a continuously musically pleasant experience. Instead their protocols used music as reward, providing the full musical stimulus only upon reaching a goal. In neither of these studies however was a comparison presented to an alternative feedback signal or to the influence of the music alone.

Finally, Yokoyama et al. (2002) presented a biofeedback system where music was controlled using the HR of a subject, and evaluated the system's usefulness towards relaxing the subject during office work. However, Yokohama et al. only compared the use of the system to a condition where the subjects worked in silence. As a result no insight was given as to whether it was the music alone that presented any benefits, or if it was its combination with biofeedback.

Future research should be motivated by the several potential benefits promised, provided only that these benefits are applicable to the context at hand. We have seen that the known effects of music at affecting the level of activation of participants can be combined with the effects of biofeedback, and result in these effects at the very least not counteracting the effect of the protocol. Because it is known humans are particularly apt at perceiving variation and detail in music, it is straightforward to expect that music also provides rich perceptual cues towards better informing the participant of their state. Over longer periods of time, we believe music should also serve towards reducing fatigue and maintaining the interest of the participant on the biofeedback signal and the cues it conveys, in comparisons to data visualization/ sonification methods, even though we did not directly test with biofeedback protocols of long duration in our current study.

\section{Conclusions}

We view the outcome of the present study as an encouraging indication that music may be used as a signal for arousal modulation using HR biofeedback. We have established that the effects of music biofeedback are stronger than use of music alone for arousal modulation. We have also established that music biofeedback is as effective in short term use as sonification biofeedback. Important implications for longer duration biofeedback use lie in our findings: a musical feedback signal has the additional positive characteristics over a sonification signal, of drawing and maintaining the person's attention, providing variation in the feedback signal which may significantly reduce the fatigue that a pure sonification signal would cause. On the other hand, the considerations one needs to take into account when using a musical signal for biofeedback are involved, and varying depending on the specific context of use. Future studies could test the validity of different physiological signals, address contexts of use other than arousal modulation, as well as try protocols of longer duration. Our findings on arousal modulation may also inform future studies that build on our contribution towards specific therapeutic interventions.

Supplementary data to this article can be found online at http:// dx.doi.org/10.1016/j.ijpsycho.2013.04.013.

\section{Acknowledgments}

We are grateful to Raphaela Groten and Tabitha Peck for their invaluable advice on experiment design and analysis. We are also grateful to the anonymous reviewers whose comments helped significantly improve an earlier draft of this paper. This research was financially supported by the INNPACTO project Melomics, funded by the Spanish Government, reference code IPT-300000-2010-010. 


\section{Appendix A. Path analysis results}

Table A.1

Path analysis results. Condition $1=$ music $2=$ biofeedback $3=$ music biofeedback Chi-squared $(6)=1.70, P=0.95$. The variables included in the model were: Task, heart rate in the experimental conditions (HR), heart rate during baseline (HRB), Log Respiration Rate (LResp) in the experimental conditions, and Log Respiration Rate during Baseline (LRespB).

\begin{tabular}{|c|c|c|c|c|}
\hline Condition & Coefficient & S.E. & $\mathrm{z}$ & $\mathrm{P}$ \\
\hline $\mathrm{HR}=$ & $+\beta 1^{*}$ LResp & & & \\
\hline 1 & 4.3 & 1.9 & 2.24 & 0.025 \\
\hline 2 & -2.2 & 1.4 & -1.63 & 0.103 \\
\hline \multirow[t]{2}{*}{3} & 0.0 & 3.3 & -0.01 & 0.994 \\
\hline & $+\beta 2 * \mathrm{HRB}$ & & & \\
\hline 1 & 0.8 & 0.1 & 7.79 & 0.000 \\
\hline 2 & 0.9 & 0.2 & 5.32 & 0.000 \\
\hline \multirow[t]{2}{*}{3} & 0.9 & 0.2 & 3.76 & 0.000 \\
\hline & + Task & & & \\
\hline 1 & -1.4 & 0.8 & -1.71 & 0.088 \\
\hline 2 & -5.7 & 1.3 & -4.52 & 0.000 \\
\hline \multirow[t]{2}{*}{3} & -4.1 & 1.3 & -3.26 & 0.001 \\
\hline & $+\beta 4^{*}$ LRespB & & & \\
\hline 1 & -2.0 & 2.0 & -0.98 & 0.327 \\
\hline 2 & 7.9 & 2.2 & 3.64 & 0.000 \\
\hline \multirow[t]{2}{*}{3} & 3.8 & 2.3 & 1.66 & 0.097 \\
\hline & $+\alpha$ & & & \\
\hline 1 & 11.2 & 3.0 & 3.71 & 0.000 \\
\hline 2 & -0.9 & 8.7 & -0.10 & 0.922 \\
\hline 3 & 6.2 & 9.2 & 0.67 & 0.500 \\
\hline LResp $=$ & Task & & & \\
\hline 1 & -0.3 & 0.1 & -4.74 & 0.000 \\
\hline 2 & -0.4 & 0.1 & -5.50 & 0.000 \\
\hline \multirow[t]{2}{*}{3} & -0.6 & 0.1 & -7.49 & 0.000 \\
\hline & $+\beta^{*}$ LRespB & & & \\
\hline 1 & 0.8 & 0.1 & 12.34 & 0.000 \\
\hline 2 & 0.9 & 0.1 & 9.64 & 0.000 \\
\hline \multirow[t]{2}{*}{3} & 1.0 & 0.1 & 8.58 & 0.000 \\
\hline & $+\alpha$ & & & \\
\hline 1 & 1.0 & 0.3 & 3.92 & 0.000 \\
\hline 2 & 1.1 & 0.3 & 3.58 & 0.000 \\
\hline 3 & 1.1 & 0.4 & 2.88 & 0.004 \\
\hline HRB & $=\mathrm{LRespB}$ & & & \\
\hline 1 & 9.2 & 3.4 & 2.66 & 0.008 \\
\hline 2 & 7.8 & 2.2 & 3.46 & 0.001 \\
\hline \multirow[t]{2}{*}{3} & 13.7 & 1.4 & 9.78 & 0.000 \\
\hline & $+\alpha$ & & & \\
\hline 1 & 43.6 & 9.3 & 4.67 & 0.000 \\
\hline 2 & 48.8 & 6.0 & 8.09 & 0.000 \\
\hline 3 & 32.6 & 4.2 & 7.71 & 0.000 \\
\hline
\end{tabular}

\section{Appendix B. Questionnaires created specifically for this experiment}

\section{B.1. Questions on experience}

1. To what extent did you feel that each condition altered your level of arousal? (Likert scale, $1-5,1=$ not at all, $3=$ neither a little nor a lot, $5=$ extremely).
1.1 First condition - increase
1.2 First condition - decrease
1.3 Second condition - increase
1.4 Second condition - decrease
1.5 Third condition - increase
1.6 Third condition - decrease

2. In the condition where your physiological activation (arousal) controlled the volume and tempo of the music, to what extent did you feel you were in control of the music? (Likert scale, 1-5, $1=$ not at all, $3=$ neither a little nor a lot, $5=$ extremely).

3. In the condition where your physiological activation (arousal) controlled the pure sound, to what extent did you feel that you were controlling the frequency of the sound? (Likert scale, 1-5, $1=$ not at all, $3=$ neither a little nor a lot, $5=$ extremely).

4. Do you feel that there are any particular circumstances in your life at this moment, which would cause you to be in a very aroused or very relaxed state? $(\mathrm{Y} / \mathrm{N})$

4.1 If so, by how much? (Likert scale, $1-5,1=$ not at all, $3=$ neither a little nor a lot, $5=$ extremely).

5. How effective at increasing your level of arousal, do you find the music we used for this purpose to have been? (Likert scale, 1-5, $1=$ not at all, $3=$ neither a little nor a lot, $5=$ extremely).

6. How effective at decreasing your level of arousal, do you find the music we used for this purpose to have been? (Likert scale, 1-5, $1=$ not at all, $3=$ neither a little nor a lot, $5=$ extremely).

7. In general, do you think that each musical piece used, was appropriate for its corresponding part of the study? (Likert scale, 1-5, $1=$ not at all, $3=$ neither a little nor a lot, $5=$ extremely).

8. Any particular reasons that you believe made it less effective than it could have been? (Open ended).

9. Is there any other issue that you would like to mention? (Open ended).

\section{B.2. Demographic and musical experience questionnaire}

1. Age

2. Gender

3. Are you taking any medication? (Y/N) If yes, please specify

4. Do you have formal studies of music? $(\mathrm{Y} / \mathrm{N})$

5. Are you a professional musician? $(\mathrm{Y} / \mathrm{N})$

6. Do you listen to music everyday? $(\mathrm{Y} / \mathrm{N})$

7. Do you feel happy, sad or euphoric depending on the musical genre? $(\mathrm{Y} / \mathrm{N})$

8. Have you experienced chills upon hearing a melody? $(\mathrm{Y} / \mathrm{N})$

9. Does music alter your emotional states? $(\mathrm{Y} / \mathrm{N})$

10. Assess your motivation to listen to music (Likert scale, 1-5, $1=$ none at all, $3=$ neither a little nor a lot, $5=$ extreme).

11. How frequently do you go to concerts or musical events? (Times per month)

12. If you play an instrument or sing: How many hours do you do so per day?

13. How frequently do you go to concerts or musical events? (Times per month)

14. Do you have experience in audio engineering/mixing or similar? $(\mathrm{Y} / \mathrm{N})$

15. Do you consider yourself a musician? $(\mathrm{Y} / \mathrm{N})$

16. Have you taught music or singing lessons? $(\mathrm{Y} / \mathrm{N})$

17. Are you receiving formal music lessons? $(\mathrm{Y} / \mathrm{N})$

18. Do you have (or will have) an official musician certificate? $(\mathrm{Y} / \mathrm{N})$

19. At what age did you start taking music lessons?

20. Are you left or right handed?

21. Do you have auditory problems? (Y/N) If yes, please specify

22. Do you have motor problems? (Y/N) If yes, please specify

23. Do you have language or speaking problems? $(\mathrm{Y} / \mathrm{N})$ If yes, please specify

24. Do you have learning problems? (Y/N) If yes, please specify

25 . Do you have visual problems that haven't been corrected? $(\mathrm{Y} / \mathrm{N})$ If yes, please specify

26. Do you have any problem that may hinder your musical perception? $(\mathrm{Y} / \mathrm{N})$ If yes, please specify

27. Have you consumed more than 2 units of alcohol in the last $6 \mathrm{~h}$ ( 2 units of alcohol $=1$ beer or 2 glasses of wine) ? $(\mathrm{Y} / \mathrm{N})$

28. Have you had coffee in the last $5 \mathrm{~h}$ ? If yes, please specify how many. 
29. For the following items, please indicate your basic preference level for the genres listed using the scale provided (Likert scale, 1-5, $1=$ strongly dislike, $3=$ neither like nor dislike, $5=$ strongly like): classical, blues, country, dance/electronic, folk, rap/hip-hop, soul/funk, religious, alternative, jazz, rock, pop, heavy metal, soundtracks.

\section{Appendix C. Task \& condition counterbalancing}

Table C.1

Participants assigned with full counterbalancing across 12 groups (G1-G12).

\begin{tabular}{lllllllllllll}
\hline Group & G1 & G2 & G3 & G4 & G5 & G6 & G7 & G8 & G9 & G10 & G11 & G12 \\
\hline $\begin{array}{c}\text { Condition } \\
\text { order }\end{array}$ & ABC & BCA & CAB & ACB & BAC & CBA & ABC & BCA & CAB & ACB & BAC & CBA \\
$\begin{array}{c}\text { Task } \\
\text { order }\end{array}$ & 1,2 & 1,2 & 1,2 & 1,2 & 1,2 & 1,2 & 2,1 & 2,1 & 2,1 & 2,1 & 2,1 & 2,1 \\
\hline
\end{tabular}

\section{References}

Ableton, 2012. Ableton - Live 8 [WWW Document]. URL http://www.ableton.com/ live-8 (accessed 6.22.12)

Ball, P., 2010. The Music Instinct: How Music Works and Why We Can't Do Without It. Bodley Head.

Bernardi, L., Porta, C., Casucci, G., Balsamo, R., Bernardi, N.F., Fogari, R., Sleight, P., 2009. Dynamic interactions between musical, cardiovascular, and cerebral rhythms in humans. Circulation 119, 3171.

Chen, Y., Sundaram, H., Rikakis, T., Ingalls, T., Olson, L., He, J., 2009. Experiential media systems-the biofeedback project. Multimedia Content Analysis 1-34.

Chiari, L., Dozza, M., Cappello, A., Horak, F.B., Macellari, V., Giansanti, D., 2005. Audiobiofeedback for balance improvement: an accelerometry-based system. IEEE Transactions on Biomedical Engineering 52, 2108-2111.

Clayton, M., Sager, R., Will, U., 2005. In time with the music: the concept of entrainment and its significance for ethnomusicology. European Meetings in Ethnomusicology, pp. 3-142.

Critchley, H.D., Wiens, S., Rotshtein, P., Öhman, A., Dolan, R.J., 2004. Neural systems supporting interoceptive awareness. Nature Neuroscience 7, 189-195.

Cui, G., Gopalan, S., Yamamoto, T., Berger, J., Maxim, P.G., Keall, P.J., 2010. Commissioning and quality assurance for a respiratory training system based on audiovisual biofeedback. Journal of Applied Clinical Medical Physics 11.

Dawson, M.E., Schell, A.M., Filion, D.L., 2000. The electrodermal system. Handbook of Psychophysiology 2, 200-223.

De l' Etoile, S.K., 2010. Neurologic music therapy. Music and Medicine 2, 78-84.

Diaz-Jerez, G., 2011. Composing with Melomics: delving into the computational world for musical inspiration. Leonardo Music Journal 13-14.

Ellis, R.J., Thayer, J.F., 2010. Music and autonomic nervous system (dys)function. Music Perception 27, 317-326.

Haas, F., Distenfeld, S., Axen, K., 1986. Effects of perceived musical rhythm on respiratory pattern. Journal of Applied Physiology 61, 1185-1191.

Hermann, T., 2008. Taxonomy and definitions for sonification and auditory display. Proceedings of the 14th International Conference on Auditory Display, pp. 1-8.

Holmes, D.S., Solomon, S., Frost, R.O., Morrow, E.F., 1980. Influence of respiratory patterns on the increases and decreases in heart rates in heart rate biofeedback training. Journal of Psychosomatic Research 24, 147-153.

Hunter, P.G., Schellenberg, E.G., 2010. Music and emotion. Music Perception, Springer Handbook of Auditory Research, 36, pp. 129-164.

Kaplan, D., 2008. Structural Equation Modeling: Foundations and Extensions. Sage Publications, Incorporated.
Khalfa, S., Isabelle, P., Jean-Pierre, B., Manon, R., 2002. Event-related skin conductance responses to musical emotions in humans. Neuroscience Letters 328, 145-149.

Khalfa, S., Roy, M., Rainville, P., Dalla Bella, S., Peretz, I., 2008. Role of tempo entrainment in psychophysiological differentiation of happy and sad music? International Journal of Psychophysiology 68, 17-26.

Koelsch, S., 2010. Towards a neural basis of music-evoked emotions. Trends in Cognitive Sciences 14, 131-137.

Kramer, G., Walker, B., Bonebright, T., Cook, P., Flowers, J.H., Miner, N., Neuhoff, J., 2010. Sonification report: status of the field and research agenda. 4

Levitin, D.J., 2006. This is Your Brain on Music. Dutton Publishing.

Levitin, D.J., Tirovolas, A.K., 2009. Current advances in the cognitive neuroscience of music. Annals of the New York Academy of Sciences 1156, 211-231.

Liu, H., 2010. Biosignal Controlled Recommendation in Entertainment Systems (PhD Dissertation)

Max/MSP, 2012. Max Cycling 74 [WWW Document]. URL http://cycling74.com/ products/max/ (accessed 6.22.12).

Mehling, W.E., Gopisetty, V., Daubenmier, J., Price, C.J., Hecht, F.M., Stewart, A., 2009 Body awareness: construct and self-report measures. PloS One 4, e5614.

Melomics, 2012. Melomics [WWW Document]. Melomics. URL http://www.melomics. $\mathrm{com} /$ (accessed 6.29.12).

Nilsson, U., 2008. The anxiety- and pain-reducing effects of music interventions: a systematic review. AORN Journal-Association of Operating Room Nurses 87, 780-807.

Nilsson, U., 2009. Caring music; music intervention for improved health.

Olson, R.P., 2003. Definitions of biofeedback and applied psychophysiology. In: Schwartz, N.M., Schwartz, M.S. (Eds.), Biofeedback. The Guilford Press, New York, pp. 27-42.

Orini, M., Bailón, R., Enk, R., Koelsch, S., Mainardi, L., Laguna, P., 2010. A method for continuously assessing the autonomic response to music-induced emotions through HRV analysis. Medical and Biological Engineering and Computing 48, 423-433.

Pan, J., Tompkins, W.J., 1985. A real-time QRS detection algorithm. IEEE Transactions on Biomedical Engineering 230-236.

Peretz, I., 2006. The nature of music from a biological perspective. Cognition 100, 1-32.

Porges, S., 1993. Body Perception Questionnaire [WWW Document]. URL http:// stephenporges.com/images/bpq.pdf (accessed 6.22.12).

Ross, A., 2007. The Rest is Noise: Listening to the Twentieth Century. Farrar Straus \& Giroux.

Salimpoor, V.N., Benovoy, M., Larcher, K., Dagher, A., Zatorre, R.J., 2011. Anatomically distinct dopamine release during anticipation and experience of peak emotion to music. Nature neuroscience 14 (2), 257-262.

Schachter, S., Singer, J., 1962. Cognitive, social, and physiological determinants of emotional state. Psychological Review 69, 379.

Simulink, 2012. Simulink - Simulation and Model-Based Design [WWW Document] URL http://www.mathworks.com/products/simulink/ (accessed 6.22.12).

SleepSense, 2012. Sleep Sense Sensors for Sleep Disorder Diagnosis [WWW Document] URL http://www.sleepsense.com/ (accessed 6.22.12).

Sloboda, J.A., 2005. Exploring the Musical Mind: Cognition, Emotion, Ability, Function. Oxford University Press.

Song, H.S., Lehrer, P.M., 2003. The effects of specific respiratory rates on heart rate and heart rate variability. Applied Psychophysiology and Biofeedback 28, 13-23.

Thomas, K., 2007. Just noticeable difference and tempo change. Journal of Scientific Psychology 2, 14-20.

Trappe, H.-J., 2010. The effects of music on the cardiovascular system and cardiovascular health. Heart 96, 1868-1871.

Tyson, P.D., 1982. The choice of feedback stimulus can determine the success of alpha feedback training. Psychophysiology 19, 218-230.

Urbaniak, G.C., Plous, S., 2011. Research Randomizer [WWW Document]. URL http://www randomizer.org/ (accessed 7.20.11)

van Boxtel, G.J., Denissen, A.J., Jäger, M., Vernon, D., Dekker, M.K., Mihajlović, V., Sitskoorn, M.M., 2012. A novel self-guided approach to alpha activity training. International Journal of Psychophysiology 83 (3), 282-294.

Yokoyama, K., Ushida, J., Sugiura, Y., Mizuno, M., Mizuno, Y., Takata, K., 2002. Heart rate indication using musical data. IEEE Transactions on Biomedical Engineering 49, 729-733.

Zatorre, R., 2005. Music, the food of neuroscience? Nature 434, 312-315.

zplane, 2011. zplane - Music Processing and Analysis Technology - élastique Time Stretching SDK [WWW Document]. URL https://www.zplane.de/index. php? page $=$ description-elastique (accessed 6.22.11). 